Artículo científico

Volumen 31(1): 129-140. Enero-abril, 2020

e-ISSN 2215-3608, doi:10.15517/am.v31i1.36776

http://www.revistas.ucr.ac.cr/index.php/agromeso

\title{
Producción y comercialización de arveja en el departamento de Nariño-Colombia ${ }^{1}$
}

\section{Pea production and commercialization in the department of Nariño-Colombia}

\author{
Francisco Javier Torres-Martínez $z^{2}$, Carlos Nelson Rivadeneira-Miranda ${ }^{3}$,́lvaro José Castillo-Marín ${ }^{4}$
}

1 Recepción: 1 de abril, 2019. Aceptación: 19 de setiembre, 2019. En este trabajo se utilizó información derivada del proyecto de investigación denominado "Aplicación del Modelo de Manufactura Esbelta para la optimización del flujo de producción de los productores de arveja (Pisum sativum L.) del departamento de Nariño”, financiado por el Sistema de Investigaciones de la Universidad de Nariño-Colombia.

2 Universidad de Nariño, Facultad de Ciencias Agrícolas, Ciudadela Universitaria-Torobajo. San Juan de Pasto, departamento de Nariño, Colombia. franjatm@hotmail.com (http://orcid.org/0000-002-9680-7145).

3 Universidad de Nariño, Facultad de Ciencias Agrícolas, Ciudadela Universitaria-Torobajo. San Juan de Pasto, departamento de Nariño, Colombia.carlosri2005@yahoo.es

4 Universidad de Nariño, Facultad de Ciencias Agrícolas, Ciudadela Universitaria-Torobajo, San Juan de Pasto, departamento de Nariño, Colombia. jacastillomarin@yahoo.es

\section{Resumen}

Introducción. En la región sur de Nariño prevalecen sistemas tradicionales de producción agrícola, sin embargo, se ha disminuido el área cosechada en cultivos transitorios de papa, maíz y frijol. Para enfrentar esta problemática se ha fortalecido la siembra de arveja, la cual se comercializa en mercados locales y regionales. El departamento de Nariño es el primer productor de arveja en Colombia, y en el 2018 se reportaron 9425 ha, con rendimiento promedio de 1,19 $\mathrm{t} \mathrm{ha}^{-1}$. Los agricultores de la zona de estudio son tradicionales y evidencian limitaciones técnicas de producción y comercialización. Objetivo. El objetivo de esta investigación fue analizar la producción y comercialización de arveja en el departamento de Nariño. Materiales y métodos. La investigación fue de carácter descriptivo y permitió establecer costos, volúmenes de producción, épocas de siembra, características del producto, precios y comercialización durante el año 2012. La población objeto del estudio incluyó productores, intermediarios municipales, mayoristas y minoristas, y líderes de asociaciones ubicadas en los municipios productores de arveja. Resultados. La variedad Andina fue la más sembrada, las pérdidas poscosecha fueron del $52 \%$, los precios presentaron variaciones por efecto de la estacionalidad de la producción, y el mayor volumen de producción fue canalizado por intermediarios. Conclusiones. La producción y comercialización de arveja en Nariño presenta dificultades que requieren de una planificación de las siembras para equilibrar los volúmenes de producción, acceder al crédito de fomento, capacitar y organizar a los agricultores y propiciar alianzas estratégicas para garantizar condiciones en la cadena de valor.

Palabras claves: costos de producción, intermediarios mayoristas, variación de precios.

\begin{abstract}
Introduction. Traditional agricultural production systems prevail in the southern region of Nariño, however, the area harvested from transitory potato, corn and beans crops has been decreased. To address this problem, pea
\end{abstract}


planting has been strengthened, which is marketed in local and regional markets. The department of Nariño is the first pea producer in Colombia, and in 2018, 9425 ha were reported, with an average yield of $1.19 \mathrm{t} \mathrm{ha}^{-1}$. The farmers in the study area are traditional and show technical limitations of production and commercialization. Objective. The objective of the investigation was to analyze the production and commercialization of peas in the department of Nariño. Materials and methods. The research was descriptive and allowed to establish costs, production volumes, planting times, product characteristics, prices, and marketing during 2012. The target population included producers, municipal intermediaries, wholesalers and retailers, and association leaders located in the pea-producing municipalities. Results. The Andean variety was the most sown, the post-harvest losses were $52 \%$, the prices vary due to the seasonality of production, and the greater volume of production was channeled through intermediaries. Conclusions. The production and commercialization of peas in Nariño presents difficulties that requiere sowing planning to balance production volumes, access development credit, train and organize farmers, and foster strategic alliances to guarantee conditions in the value chain.

Keywords: production costs, wholesale intermediaries, price variation.

\section{Introducción}

Un problema en la zona sur del departamento de Nariño se relaciona con la disminución de áreas cosechadas en cultivos transitorios, la cual presentó una marcada reducción hasta el año 2005 (SAD, 2005); los cultivos más afectados fueron papa, zanahoria, arveja, maíz, cebada y trigo, como consecuencia de la influencia de las políticas de apertura económica, problemas fitosanitarios y climáticos; no obstante, en los últimos quince años, el cultivo de la arveja presenta una perspectiva positiva y las áreas sembradas en la zona Andina de Nariño se han multiplicado sustancialmente.

En el año 2017 en el departamento de Nariño se sembraron 7290 ha, mientras que en el año 2018 se reportaron $9425 \mathrm{ha}$, con un rendimiento promedio de $1,19 \mathrm{t} \mathrm{ha}^{-1}$; que la ubica como la zona con mayor producción de arveja en Colombia (FENALCE, 2018).

El cultivo de arveja (Pisum sativum L.) en Colombia es la segunda leguminosa en importancia y constituye un factor estabilizador de la economía de los pequeños productores de la zona Andina; además, contribuye a la seguridad alimentaria y de empleo rural, por la cantidad de mano de obra que requieren las labores culturales del cultivo (Casanova et al., 2012). El consumo de arveja ha alcanzado en diferentes segmentos de la población, principalmente urbana, un alto nivel de exigencia, donde las características físicas del producto constituyen un factor diferencial e indispensable; por tanto, la decisión de compra se basa en el valor agregado.

El departamento de Nariño es uno de los principales proveedores de arveja fresca, especialmente en los mercados del departamento del Valle del Cauca, eje cafetero y centro del país, lo que genera una alternativa de producción muy atractiva para los productores nariñenses. Los clientes mayoristas y minoristas se han visto obligados a replantear sus modelos de venta y operaciones, para ofrecer presentaciones y precios que se adapten al gusto y alcance de los diferentes tipos de consumidores, y eliminar en su operación la mayor cantidad de procesos, para reducir el volumen de sus inventarios y optar por hacer pedidos con características específicas de composición, surtido y empaque, que pueden ofrecer sus proveedores en un corto margen de tiempo.

La referencia de modelos implementados con éxito en diferentes sistemas agrícolas, pueden ser adaptados a las condiciones particulares de la producción de arveja y permite la transición a métodos de producción más eficientes y competitivos; por ejemplo, el Modelo de Manufactura Esbelta (MME), es una filosofía de producción 
que enfatiza en la especificación de valor definido por el cliente final y la minimización de los recursos (incluido el tiempo), empleados en varias actividades de la empresa. Incluye la identificación y eliminación de actividades que no le añaden valor a la producción, la gestión de la cadena de suministro y la entrega oportuna a los clientes (Gaither y Frazier, 2002). Otra referencia, es el Mapeado de Flujo de Valor (MFV), como una técnica reciente que viene a dar respuesta a las necesidades planteadas por los productores, para desarrollar cadenas de valor más competitivas, sólidas, eficientes y flexibles para afrontar las dificultades de la economía actual (Serrano, 2007). El fomento de estas actividades del sector primario no solo incluyen paquetes tecnológicos y sistemas de comercialización más eficientes, sino que se complementan con la financiación de capital destinado a la siembra de cultivos transitorios y construcción de infraestructura para el almacenamiento de productos agropecuarios; para ello, el Estado colombiano ofrece programas de fomento a través del Fondo para el Financiamiento del Sector Agropecuario (FINAGRO), que incluye un portafolio de servicios con líneas de crédito a tasas de interés inferiores a las que ofrece el sector bancario tradicional (FINAGRO, 2018).

El cultivo de arveja representa una opción económica rentable para los pequeños agricultores de la zona Andina, situación que confirman Peñaranda y Molina (2011), quienes aseveran que el cultivo es clave para el desarrollo de la economía nacional por sus bajos costos y fácil producción. El comportamiento de la siembra de arveja en la zona de estudio (Figura 1), coincide con los reportes del Consolidado Agropecuario de Nariño (CCI, 2008), que destacaron un incremento sustancial el área sembrada, principalmente con las variedades San Isidro y Andina. Esta situación también la corroboran Checa y Rodríguez (2015), y aseguraron que las variedades más utilizadas en el departamento de Nariño corresponden a Andina, San Isidro, Sureña y Sindamanoy. De igual manera, Burbano et al. (2018), afirmaron que la variedad Andina presenta mayores rendimientos, y el estudio confirmó que los productores en siete municipios se inclinaron por la siembra de variedades de arveja de mayor rendimiento.

Esta investigación tuvo como objetivo analizar la producción y comercialización de la arveja en el departamento de Nariño.

\section{Materiales y métodos}

La investigación acudió al método inductivo-deductivo, observacional y analítico aplicado a una realidad observada en la zona de producción durante el año 2012. Adicionalmente, se consultó, analizó y confrontó información especializada proveniente de instituciones y personas naturales dedicadas al rubro en estudio.

\section{Zona de estudio}

Esta investigación se realizó en los municipios de Ipiales, Pupiales, Córdoba, Potosí, Carlosama, Puerres y Gualmatán, ubicados en la zona Andina, al sur del departamento de Nariño, Colombia. Las siete localidades se encuentran a una altura promedio de $2950 \mathrm{msnm}$, temperatura de $10{ }^{\circ} \mathrm{C}$ y precipitación media de $1200 \mathrm{~mm}$ anuales.

\section{Recolección de información}

La investigación recopiló información de: agricultores, intermediarios municipales, que comercializan la arveja a nivel intermunicipal, intermediarios mayoristas, se refiere a los que comercializan hacia otros departamentos, intermediarios minoristas son los que comercializan en las plazas de mercado, y líderes de asociaciones de los siete municipios productores de arveja. La recolección de la información primaria se llevó a cabo mediante el método probabilístico y se empleó la ecuación 1 (Hernández et al., 2014) para determinar el número de encuestas. 


\section{$\mathbf{n}=\frac{\mathbf{N} \times \mathbf{Z} \times \mathbf{P} \times \mathbf{Q}}{\left(\boldsymbol{N} \times \boldsymbol{E}^{\mathbf{2}}\right)+\boldsymbol{Z} \times \boldsymbol{P} \times \boldsymbol{Q}} \quad$ Ecuación 1}

Donde:

$\mathrm{N}=$ población.

$\mathrm{Z}=$ coeficiente de confianza.

$E=$ error de estimación.

$\mathrm{P}=$ cliente a favor.

$\mathrm{Q}=$ cliente en contra.

$\mathrm{n}=$ muestra.

Las encuestas incluyeron un cuestionario de preguntas cerradas y se efectuaron de manera directa. La información se organizó, tabuló y analizó mediante cuadros y tablas con el programa de Microsoft Excel. El número de participantes en el estudio y las variables objeto de análisis con sus respectivos indicadores, se observan en el Cuadro 1.

Cuadro 1. Encuestados, número de encuestas, variables e indicadores para la producción y comercialización de arveja (Pisum sativum L.), en los municipios de Ipiales, Pupiales, Córdoba, Potosí, Carlosama, Puerres y Gualmatán, del departamento de Nariño. Colombia. 2012.

Table 1. Respondents, number of surveys, variables and indicators for pea (Pisum sativum L.) production and marketing in the municipalities of Ipiales, Pupiales, Cordoba, Potosi, Carlosama, Puerres, and Gualmatan of the department of Nariño, Colombia. 2012.

\begin{tabular}{|c|c|c|c|}
\hline Encuestados & Encuestas & Variables & Indicadores \\
\hline Agricultores & 260 & $\begin{array}{l}\text { Costos de producción } \\
\text { variedades utilizadas } \\
\text { Época de siembra } \\
\text { Rendimiento } \\
\text { características del producto } \\
\text { Actividades de poscosecha, } \\
\text { Precios de venta } \\
\text { Plazo de pago. }\end{array}$ & $\begin{array}{l}\text { US ha }^{-1} \\
\text { Denominación } \\
\text { Meses } \\
\mathrm{kg} \mathrm{ha}^{-1} \\
\text { Color, tamaño } \\
\text { Actividades } \\
\text { US bulto } \\
\text { Días }\end{array}$ \\
\hline Intermediarios municipales & 20 & $\begin{array}{l}\text { Precios de compra y venta } \\
\text { Volúmenes de compra } \\
\text { Características del producto } \\
\text { Acuerdo de precio }\end{array}$ & $\begin{array}{l}\text { US bulto }^{-1} \\
\text { Bultos } \\
\text { Color, tamaño } \\
\text { Opciones }\end{array}$ \\
\hline Intermediarios mayoristas & 32 & $\begin{array}{l}\text { Precios de compra y venta } \\
\text { Volúmenes de compra } \\
\text { características del producto } \\
\text { Acuerdo de precio }\end{array}$ & $\begin{array}{l}\text { US bulto }^{-1} \\
\text { Bultos } \\
\text { Color, tamaño } \\
\text { Opciones }\end{array}$ \\
\hline Intermediarios minoristas & 22 & $\begin{array}{l}\text { Precios de compra y venta } \\
\text { volúmenes de compra } \\
\text { Características del producto } \\
\text { Acuerdo de precio }\end{array}$ & $\begin{array}{c}\text { US } \\
\text { Bultos } \\
\text { Color, tamaño } \\
\text { Opciones }\end{array}$ \\
\hline Líderes de asociaciones & 7 & $\begin{array}{l}\text { Número de asociados } \\
\text { Capacitación } \\
\text { Acceso al crédito agrícola } \\
\text { Características del crédito } \\
\text { Aspectos legales }\end{array}$ & $\begin{array}{l}\text { Número } \\
\text { No. de cursos } \\
\text { No. usuarios } \\
\text { Interés, plazo } \\
\text { Personería }\end{array}$ \\
\hline
\end{tabular}


Las fuentes de información secundaria contienen antecedentes de producción y comercialización de la arveja, estudios de mercado de productos agrícolas, aspectos geográficos de los municipios y, entidades públicas y privadas relacionadas. Al final, se plantean recomendaciones para lograr un flujo continuo y eficiente de producto para elevar el nivel de competitividad los productores en el mercado.

\section{Resultados}

\section{Producción}

El $50 \%$ de los agricultores reportaron que la variedad de arveja más utilizada para la siembra en la zona de estudio correspondió a la denominada Andina. Por sus características fue la más aceptada por los agricultores y comerciantes, porque el grano es más brillante y más resistente al transporte. En segundo lugar, se ubicó la variedad San Isidro con el $35 \%$, y los agricultores la prefirieron porque obtienen vainas desde los primeros entrenudos en el tallo, la vaina es más larga y facilita la extracción de semillas para las próximas siembras. También reportan otras variedades, como la Piquinegra con el $7 \%$ y Sindamanoy con $1 \%$ (Figura 1).

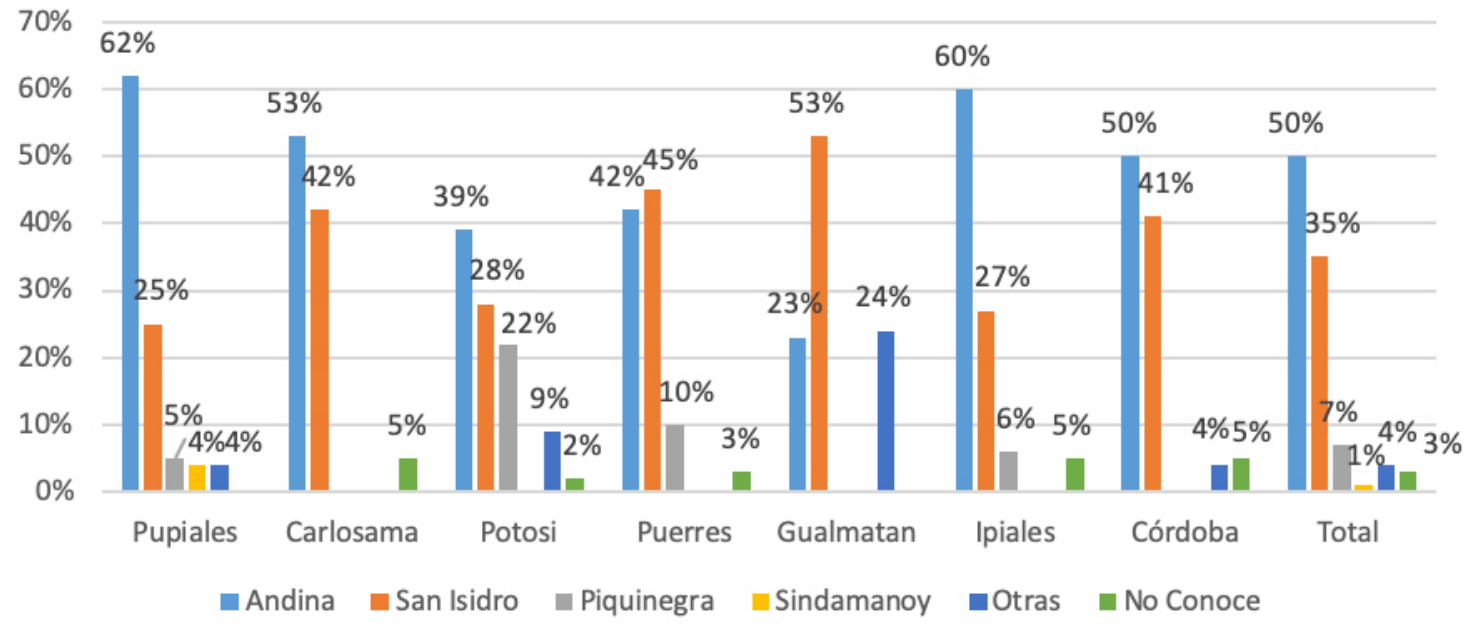

Figura 1. Variedades de arveja (Pisum sativum L.), sembradas en siete municipios de la zona sur del departamento de Nariño: Ipiales, Pupiales, Córdoba, Potosí, Carlosama, Puerres y Gualmatán. Colombia. 2012.

Figure 1. Pea (Pisum sativum L.) varieties planted in seven municipalities in the southern area of the department of Nariño: Ipiales, Pupiales, Cordoba, Potosi, Carlosama, Puerres, and Gualmatan. Colombia. 2012.

\section{Costos de producción promedio por hectárea}

Los costos totales promedio de producción del cultivo de arveja en la zona aumentaron a U\$ 3381,08 , los cuales fueron similares a los reportados por el Sistema de Información de Precios del Sector Agropecuario (SIPSA); no obstante, presentaron diferencias mínimas del 1,58 \% (Cuadro 2). Del total de costos, el 86,5\% correspondieron a costos directos y el $13,50 \%$ a costos indirectos. 
Cuadro 2. Costos de producción promedio de arveja (Pisum sativum L.), en siete municipios de la zona sur del departamento de Nariño: Ipiales, Pupiales, Córdoba, Potosí, Carlosama, Puerres y Gualmatán. Colombia. 2012.

Table 2. Average production costs of pea (Pisum sativum L.), in seven municipalities of the southern area of the department of Nariño: Ipiales, Pupiales, Cordoba, Potosi, Carlosama, Puerres, and Gualmatan. Colombia. 2012.

\begin{tabular}{ccccc}
\hline & Zona de estudio & SIPSA & Diferencia & \multicolumn{2}{c}{ Diferencia } \\
\cline { 2 - 5 } Costos/ ha & U\$* & U\$* & U\$* & \% \\
\hline Directos & 2924,64 & 2975,11 & 50,47 & 1,73 \\
Indirectos & 456,44 & 459,46 & 3,02 & 0,66 \\
Totales & 3381,08 & 3434,58 & 53,49 & 1,58 \\
\hline
\end{tabular}

* La tasa de cambio promedio del dólar histórico del año 2012 en Colombia fue de COL\$ 1,797.79/UD\$. Fuente: dolarweb (2012) / * The average exchange rate of the historical dollar of the year 2012 in Colombia was COL\$1,797.79/UD\$. Source: dolarweb.com (2012).

En los costos directos del cultivo de arveja se destacaron diversas actividades por sus montos; por ejemplo, el tutorado ${ }^{4}$ representó el 22,82 \%, luego se sitúan las labores culturales con el 19,78 \%, la cosecha con 12,11 \% y los costos derivados de los insumos agroquímicos y semilla, entre otros (Cuadro 3).

Cuadro 3. Costos promedio directos por hectárea de arveja (Pisum sativum L.), en siete municipios de la zona sur del departamento de Nariño: Ipiales, Pupiales, Córdoba, Potosí, Carlosama, Puerres y Gualmatán. Colombia. 2012.

Table 3. Direct average costs by hectare of peas (Pisum sativum L.), in the seven municipalities of the southern area of the department of Nariño: Ipiales, Pupiales, Cordoba, Potosi, Carlosama, Puerres, and Gualmatan. Colombia. 2012.

\begin{tabular}{|c|c|c|c|c|c|}
\hline & Ítem & Valor total US* & Cantidad & Unidad & $\%$ \\
\hline 1 & Preparación del terreno & 100,11 & 6 & $\mathrm{~h} \mathrm{Maq}^{-1}$ & 3,42 \\
\hline 2 & Adecuación del terreno & 50,61 & 7 & Jornales & 1,73 \\
\hline 3 & Siembra & 86,76 & 12 & Jornales & 2,97 \\
\hline 4 & Labores culturales & 578,42 & 80 & Jornales & 19,78 \\
\hline 5 & Aplicación de insumos & 151,84 & 21 & Jornales & 5,19 \\
\hline 6 & Cosecha & 354,28 & 49 & Jornales & 12,11 \\
\hline 7 & Semilla & 160,18 & 36 & $\mathrm{~kg}$ & 5,48 \\
\hline 8 & Enmienda & 11,12 & 2 & Bultos & 0,38 \\
\hline 9 & Fertilizante orgánico & 5,56 & 4 & Bultos & 0,19 \\
\hline 10 & Fertilizante edáfico & 266,96 & 6 & Bultos & 9,13 \\
\hline 11 & Fertilizantes foliares & 82,04 & 5,9 & 1 & 2,8 \\
\hline 12 & Fungicidas & 222,47 & - & $\mathrm{kg}$ y 1 & 7,61 \\
\hline 13 & Insecticidas & 33,37 & 1 & 1 & 1,14 \\
\hline 14 & Herbicidas & 22,25 & 1 & 1 & 0,76 \\
\hline 15 & Coadyudantes & 20,02 & 2 & 1 & 0,68 \\
\hline 16 & Empaque & 111,23 & Global & Global & 3,8 \\
\hline \multirow[t]{2}{*}{17} & Tutorado & 667,41 & Global & Global & 22,82 \\
\hline & & 2924,64 & 227 & & 100 \\
\hline
\end{tabular}

* La tasa de cambio promedio del dólar histórico del año 2012 en Colombia fue de COL\$1797.79/UD\$. dolarweb.com (2012) / * The average exchange rate of the historical dollar of the year 2012 in Colombia was COL\$ 1797.79/UD\$. dolarweb.com (2012).

$4 \quad$ El tutorado consiste en ofrecer soporte al cultivo de arveja, mediante estacas de madera para que las plantas puedan crecer adecuadamente, y evitar que las vainas tengan contacto con el suelo, y con ello obtener mayor producción y reducir pérdidas por pudrición. 


\section{Épocas de producción}

El 86,93\% de los agricultores sembraron arveja motivados por los precios de venta. Por lo general, las siembras se realizaron entre los meses de enero a marzo, y la época de mayor cosecha se ubicó entre los meses de mayo y agosto; el mes de mayo fue el que registró mayor cosecha con el $24 \%$, luego se ubicaron los meses de junio con $22 \%$ y julio con $17 \%$ (Figura 2).

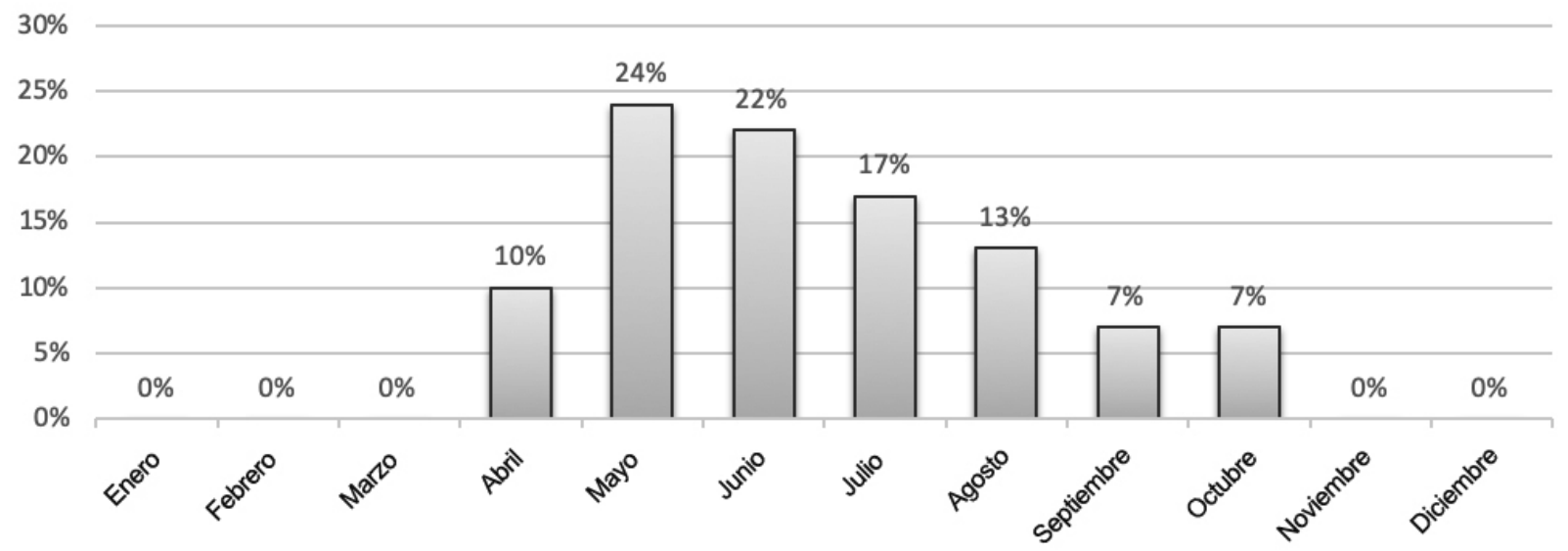

Figura 2. Época de cosecha de arveja (Pisum sativum L.), en siete municipios de la zona sur del departamento de Nariño: Ipiales, Pupiales, Córdoba, Potosí, Carlosama, Puerres y Gualmatán. Colombia. 2012.

Figure 2. Harvesting time of pea (Pisum sativum L.), in seven municipalities of the southern area of the department of Nariño: Ipiales, Pupiales, Cordoba, Potosi, Carlosama, Puerres, and Gualmatan. Colombia. 2012.

\section{Destino de la producción y porcentajes comercializados}

El mayor volumen de la producción de arveja en la zona de estudio se vendió directamente en la fincas de los agricultores a los intermediarios municipales, mayoristas y minoristas; en su orden, otros volúmenes de producción se comercializaron en las plazas de mercado de Ipiales, a través de cooperativas, en lotes de terreno sin cosechar, a mercados de las ciudades de Bogotá, Medellín y Cali, y un pequeño volumen al mercado de la ciudad de Pasto. El destino y porcentajes comercializados de arveja en la zona de estudio se muestra en la Figura 3.

\section{Determinación de precios de compra y venta}

La determinación de los precios de compra y venta de la arveja en la zona de estudio se establecieron mediante regateo, y estos presentaron variaciones sustanciales, suscitadas principalmente por la estacionalidad de la producción (Figura 2). El precio de la arveja fresca en un día puede variar entre USD \$ 36,15 bulto $^{-1}$ a USD \$ 41,72 bulto $^{-1}$, lo que significa variaciones por bulto de hasta $15 \%$. También se pudo determinar que los productores de arveja no disponían de información confiable y oportuna sobre volúmenes producidos y condiciones de mercado; de allí que los mayoristas posean mayores posibilidades de fijar los precios de compra y venta. 


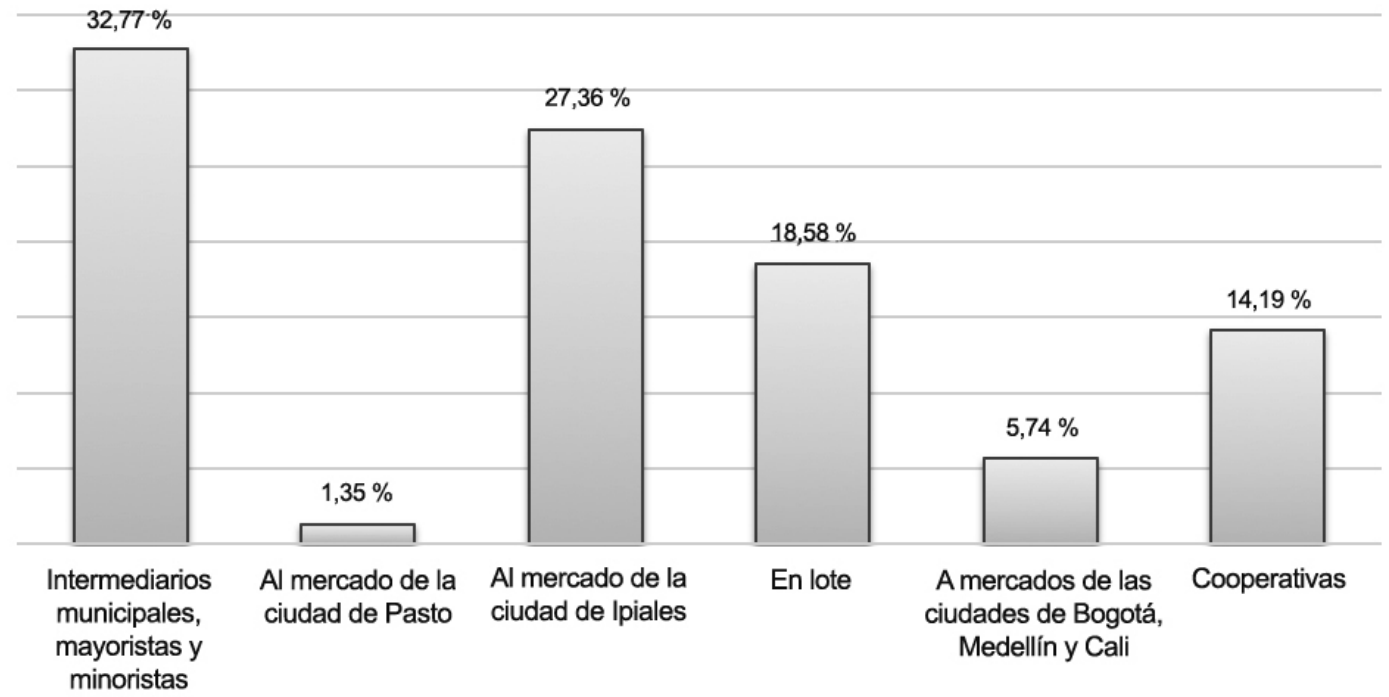

Figura 3. Porcentajes de arveja comercializados (Pisum sativum L.) en siete municipios de la zona sur del departamento de Nariño: Ipiales, Pupiales, Córdoba, Potosí, Carlosama, Puerres y Gualmatán. Colombia. 2012.

Figure 3. Percentages of commercialized pea (Pisum sativum L.), in seven municipalities of the southern area of the department of Nariño: Ipiales, Pupiales, Cordoba, Potosi, Carlosama, Puerres, and Gualmatan. Colombia. 2012.

\section{Empaque y peso promedio por bulto}

La arveja en la zona de estudio se comercializó en un $85 \%$ en empaques de polietileno y el $15 \%$ restante utilizaron empaques de fibra natural (fique). La utilización de estos últimos, permite el muestreo o inspección del producto con el empleo de un chuzo ${ }^{5}$, y la perforación de los empaques no ocasiona perdidas del producto. El peso promedio convenido entre productores y comercializadores de arveja fue de $52 \mathrm{~kg}^{\text {bulto }}{ }^{-1}$.

\section{Clasificación, selección y almacenamiento}

En la zona de estudio la totalidad de los intermediarios municipales y mayoristas (100\%) realizaron actividades de clasificación y desgrane de vainas, y la selección de granos de arveja. La arveja cosechada en vaina se inspeccionó visualmente y se clasificó de manera manual, y constituyó un método eficiente para extraer producto deteriorado con manchas o con exceso de humedad; posteriormente, el desgrane se realizó manualmente. Para esta labor se requirió de un trabajador por bulto de arveja.

Con relación al almacenamiento, el 97 \% de los productores manifiestaron no hacerlo, en razón a que la arveja se negocia con la condición de entregarla el mismo día de la cosecha, debido a que el producto es perecedero. El $3 \%$ restante almacenó el producto en cuartos refrigerados. A nivel de intermediarios municipales y mayoristas,

5 Instrumento fabricado en acero inoxidable de $30 \mathrm{~cm}$ de longitud y diámetro de $2,5 \mathrm{~cm}$, que se utiliza para tomar muestras de arveja. 
el $55 \%$ almacenaron la arveja desgranada en cuartos refrigerados, mientras que el $45 \%$ restante no disponiá de infraestructura adecuada para esta actividad.

El $64 \%$ de los distribuidores minoristas compraron la arveja en centros de acopio, situación que según ellos, disminuye riesgos de pérdida por manejo poscosecha; y el $34 \%$ compraron la arveja directamente en las fincas de los agricultores.

\section{Financiación y asociación}

En general, el $76 \%$ de los agricultores encuestados recurrió al crédito para efectuar la siembra de arveja y el $24 \%$ restante no utilizó este instrumento financiero. Del total de agricultores que recurrieron a la financiación, solo el $20 \%$ correspondió al crédito de fomento del sistema FINAGRO, el cual se canalizó principalmente por el Banco Agrario $^{6}$, en las ciudades de Pasto, Ipiales y Túquerres. Las líneas de crédito FINAGRO tuvieron condiciones favorables en cuanto al plazo y tasa de interés; no obstante, su reducida utilización obedece, según manifiestan los agricultores, al exceso de trámites bancarios y a la dificultad para cumplir las condiciones legales que exigen las entidades financieras. Mientras que el $80 \%$ restante recurrió a otras formas de financiación más costosas, como los préstamos personales; sin embargo, aducieron que son más expeditos y con menores trámites.

Con relación a los intermediarios mayoristas, no utilizaron crédito y para realizar las actividades de comercialización de la arveja acudieron a las reservas financieras propias en efectivo, las cuales han capitalizado a través del tiempo.

Las asociaciones de productores sí utilizaron el crédito del Banco Agrario, representado en capital de trabajo destinado a cubrir costos de comercialización de arveja. Entre otras asociaciones que utilizaron financiación, se destacaron la Sociedad Agraria Productora, Comercializadora y Transformadora de Arveja (SOPROCOTAR), Asociación de Productores de Chaguaipe (ASOCHAGUAIPE) y la Asociación de Productores de Arveja de la Cofradia (ASOCOFRADIA).

Con respecto a la intención de los agricultores de asociarse para comercializar la arveja, el $58 \%$ estuvo de acuerdo en organizar cooperativas de productores, y adujeron que estas empresas podrían mejorar las condiciones de negociación y garantizar precios más estables, siempre y cuando exista una eficiente administración. Por su parte, el $42 \%$ restante no estuvo de acuerdo con asociarse y argumentaron experiencias negativas con grupos solidarios creados anteriormente en la zona de estudio.

\section{Pérdidas poscosecha}

En la zona del estudio se registraron pérdidas poscosecha, entre las más significativas las causadas por exceso humedad y manchas en la vaina, producidas por hongos como antracnosis (Colletotrichum, Gloeosporium, o la especie Coniothyrium fuckelii) y botrytis (Botryotinia fuckeliana (de Bary) Whetzel). Otras pérdidas frecuentes de tipo físico, son causadas por inadecuados sistemas de almacenamiento del grano. En la zona de estudio se logró establecer que cuando el producto cosechado presentó buen aspecto, de un bulto de arveja en vaina con peso de $52 \mathrm{~kg}$, se obtuvieron $25 \mathrm{~kg}$ de grano fresco de arveja, lo que equivale al $48 \%$ de rendimiento. En casos extremos, cuando la arveja en vaina presentó deterioros mayores al $50 \%$, el producto fresco en grano apto para comercializar se puede reducir a $20 \mathrm{~kg}$ bulto $^{-1}$.

6 El Banco Agrario de Colombia es una entidad financiera estatal fundada en 1999, la cual, reemplazó a la antigua Caja de Crédito Agrario. Tiene oficinas en todo el territorio colombiano y ofrece servicios, principalmente de crédito de fomento, en localidades pequeñas y con dedicación agropecuaria, donde no tienen cobertura otras entidades financieras privadas. 


\section{Formas de pago}

La forma de pago a los agricultores de arveja por parte de los intermediarios, se estableció en dos entregas, la primera correspondió al $50 \%$ después de entregar el producto, y el $50 \%$ restante se pagó posteriormente. Esta situación, a juicio de los agricultores, no es favorable, ya que se presentaron incumplimientos de pago por parte de los intermediarios municipales y mayoristas, los cuales adujeron pérdidas ocasionadas por los rechazos del producto en los mercados.

\section{Discusión}

Con relación a los costos de producción, una de las actividades más relevantes fue el tutorado del cultivo, el cual representó el 22,83 \% (Cuadro 2); mientras que Checa y Rodríguez (2015), afirman que los egresos asignados a esta actividad fueron mayores y representaron cerca del $52 \%$ de los costos; a su vez, Peñaranda y Molina (2011), aseguraron que el tutorado representó un aproximado del $15 \%$. La diferencia en los costos del tutorado, para uno y otro caso, obedecen a que en la zona de estudio, la mayoría de agricultores son minifundistas y la tecnología para la producción es tradicional, por lo tanto, los costos del tutorado son más bajos; mientras que los costos de tutorado para productores con mayor grado de tecnología son más elevados.

La producción en la zona de estudio se concentró en determinadas épocas del año, esta situación originó sobreoferta y con ello, la consecuente disminución de precios (Figura 2). A lo anterior, se suma la escasa planificación de siembras y la incipiente organización de los productores de arveja, situación que favorece la fluctuación de precios de compra y venta. El estudio confirmó la presencia de varios intermediarios en la cadena de comercialización del producto, situación que corroboró Torres (2010), quien aseguró que los países latinoamericanos se caracterizan por la excesiva intermediación comercial. Por su parte, Figueroa et al. (2012), concluyeron que los intermediarios que comercializaron productos agrícolas precederos poseían habilidad negociadora que posibilitó pactar las condiciones de oferta y demanda y fijaron unilateralmente los precios en favor de sus intereses.

Los precios bajos de venta también se mencionaron en el Boletín de Precios del Departamento Administrativo Nacional de Estadísticas (DANE, 2013), que a finales de 2012 registraron una reducción en los precios de la arveja verde en vaina a causa de una mayor producción en la Sabana de Bogotá y Nariño (DANE, 2013). La comercialización y el mercadeo fueron los problemas más importantes que enfrentó el agricultor, por la necesidad de entregar las cosechas al primer comprador y al precio que este le ofrezca (Coral et al., 2012).

Las pérdidas poscosecha verificadas en la zona de estudio pudieron superar el $50 \%$ y la mayoría tuvo que ver con la pudrición producida por hongos; este escenario lo corroboraron Valencia et al. (2012), quienes aseguraron que las pérdidas se presentan en la etapa de cosecha, debido a las precipitaciones que favorecen el desarrollo de patógenos fungosos que dañan los órganos aéreos de la planta como hojas, tallos y vainas. Con relación al crédito de fomento, constituye una opción real en la zona de estudio y se puede fortalecer el acceso por parte de los agricultores, mediante un mayor apoyo de instituciones públicas; al respecto, Cano (2006), sugiere que el Estado puede amparar los créditos agropecuarios mediante garantías.

\section{Conclusiones}

La mayoría de cultivadores de arveja en la zona de estudio eran minifundistas, la tecnología era tradicional, el volumen de producción individual era bajo y su participación en el mercado no era significativa. Los precios estaban influenciados por la estacionalidad de la producción y por los intermediarios mayoristas. Las pérdidas 
poscosecha eran importantes y afectaban los ingresos de los productores. La mayoría de los agricultores deseaban capacitarse y constituir cooperativas o empresas comercializadoras para el mercadeo de arveja, sin embargo, experiencias negativas del pasado constituían un serio obstáculo para incentivar nuevas asociaciones.

Las recomendaciones tenían que ver con estrategias de planificación de siembras para equilibrar volúmenes de producción a lo largo del año y con ello, disminuir los precios cíclicos. Capacitar a los agricultores para que un mayor número acceda al crédito de fomento individual para siembra de arveja, a través del sistema de crédito estatal FINAGRO y capacitar a líderes para la conformación y fortalecimiento de las asociaciones de productores y grupos asociativos para direccionar precios de venta más competitivos.

\section{Literatura citada}

Burbano, E., J.J. Domínguez, y O.E. Checa. 2018. Efecto de cinco densidades de siembra en líneas de arveja Pisum sativum L. con el gen mutante. Invest. Agrar. 20(1):22-29. doi:10.18004/investig.agrar.2018.junio.22-29

Cano, C. 2006. Después del TLC ¿qué? Intermedio Editores, Bogotá, COL.

Casanova, L., J. Solarte, y O. Checa. 2012. Evaluación de cuatro densidades de siembra en siete líneas promisorias de arveja arbustiva (Pisium sativum L.). Rev. Cienc. Agric. 29(2):129-140.

Checa, O., y M. Rodríguez. 2015. Resistencia a oídio (Erysiphe polygoni) y rendimiento en arveja afila (Pisum sativum L.). Temas Agrar. 20(2):58-71.

Coral, L., F. Torres, y B. Yépez. 2012. Estudio de mercado para la comercialización de uchuva Physalis peruviana L. en Nariño. Rev. Cienc. Agric. 29(1):88-98.

DANE (Departamento Administrativo Nacional de Estadística). 2013. Precios mayoristas. Boletín Semanal No. 30. DANE, COL. https://www.dane.gov.co/files/investigaciones/agropecuario/sipsa/Semana_5ene_11ene_2013.pdf (consultado 9 ene. 2018).

CCI (Corporación Colombiana Internacional). 2008. Consolidado agropecuario 2008. Ministerio de Agricultura, Corporación Colombia Internacional, y Secretaría de Agricultua y Medio Ambiente, Pasto, COL.

dolarweb. 2012. Dólar histórico en Colombia del año 2012. dolarweb, COL. https://dolar.wilkinsonpc.com.co/dolar-historico/ dolar-historico-2012.html (consultado 15 feb. 2019).

FENALCE (Federación Nacional de Cultivadores de Cereales y Leguminosas). 2018. Área, producción y rendimiento cereales y leguminosas 2018 A. FENALCE, COL. http://www.fenalce.org/alfa/dat_particular/ar/ar_25195_q_APR_2018_A_Abril. pdf (consultado 17 feb. 2019).

Figueroa, D., D. Rosas, y F. Torres. 2012. Comercialización de papa de las variedades Diacol, capiro, parda pastusa Solunum tuberosum L. y amarilla Solanum phureja, en tres corregimientos del municipio de Pasto. Rev. Cienc. Agríc. 29(1):16-28.

FINAGRO (Fondo para el Financiamiento del Sector Agropecuario). 2018. Portafolio de servicios de crédito. FINAGRO, Bogotá, COL.

Gaither, N., and G. Frazier. 2002. Operations management. $9^{\text {th }}$ ed. Prentice Hall, OH, USA.

Hernández, R., C. Fernández, y P. Baptista. 2014. Metodología de la investigación. 6 ${ }^{\text {ta }}$ ed. El Estado de Jalisco Peródico Oficial, MEX. https://periodicooficial.jalisco.gob.mx/sites/periodicooficial.jalisco.gob.mx/files/metodologia_de_la_ investigacion_-_roberto_hernandez_sampieri.pdf (consultado 29 ago. 2019).

Peñaranda, G., y D. Molina. 2011. La producción de arveja (Pisum sativum) en la vereda Monteadentro, Provincia de Pamplona, Norte de Santander. Face 7(11):43-56. 
SAD (Secretaría de Agricultura Departamental). 2005. Informe estadístico agropecuario. Imprenta Departamental, San Juan, Pasto, COL.

Serrano, I. 2007. Análisis de la aplicabilidad de la técnica Value stream mapping en el rediseño de sistemas productivos. Tesis Dr., Universidad de Girona, Girona, ESP.

Torres, G. 2010. Distribución de alimentos. Mercados y políticas sociales. Universidad Nacional Autónoma de México, MEX.

Valencia, A., Y. Timaná, y O. Checa. 2012. Evacuación de 20 líneas de arveja (Pisum sativum L.) y su reacción al complejo de Ascochyta. Rev. Cienc. Agríc. 29(2):39-52. 